\title{
Communications
}

\section{An Open Architecture Patient Monitoring System Using Standard Technologies}

Péter Várady, Zoltán Benyó, and Balázs Benyó

\begin{abstract}
Computer-aided bedside patient monitoring is applied in areas where real-time vital function analysis takes place. Modern bedside monitoring requires not only the networking of bedside monitors with a central monitor but also other standard communication interfaces. In this paper, a novel approach to patient monitoring is introduced. A patient monitoring system was developed and implemented based on an existing industry standard communication network, using standard hardware components and software technologies. The open architecture system design offers scalability, standard interfaces, and flexible signal interpretation possibilities.
\end{abstract}

Index Terms-Artificial patient, fieldbus, medical communication, open architecture, patient monitoring.

\section{INTRODUCTION}

Patient monitors are the most important diagnostic devices in the critical care units (CCUs) of hospitals, providing continuous display and interpretation of the patient's vital functions. The rapid evolution of electronics and information technology is resulting in more powerful bedside patient monitors capable of complex biosignal processing and interpretation and usually equipped with some specialized communication interface.

During the last decade, centralized patient monitoring systems were installed in the majority of critical care facilities. Centralized patient monitoring provides the networking of several bedside patient monitors with a central monitoring station (central monitor) [1].

One of the most recent challenges in the field of critical care is the integration of patient monitoring into the hospital information system (HIS). HIS is the base element of the modern hospital information infrastructure, frameworking each health care department from CCU through radiology to billing. Obviously the integration of patient monitors depends on the availability of an appropriate standard that describes the information interface between the monitoring device and the HIS mainframe. The patient data management system (PDMS) is a component of HIS dedicated to the standardized interaction with critical care monitors. The PDMS cyclically collects, validates, and provides automatic charting of vital parameters [1].

The emergence of Internet technologies and telemedicine also opens new scales and makes new demands on patient monitoring [2], [3]. Home-care monitoring offers faster, more effective, and cost-saving rehabilitation and mobilization of patients. Telecare monitors can provide both holter and on-line, noninvasive monitoring of vital functions.

The increasing demands outlined above present new requirements for patient monitors, whereby two paradigms play the most important

Manuscript received June 14, 2000; revised June 26, 2001. This work was supported by the European Union under INCO Copernicus Grant 960161 and by the Hungarian National Research Fund under Grants OTKA T029830 and OTKA F029739.

P. Várady and Z. Benyó are with the Department of Control Engineering and Information Technology, Budapest University of Technology and Economics, H-1117 Budapest, Hungary (e-mail: varady@iit.bme.hu; benyo@iit.bme.hu).

B. Benyó is with the Department of Informatics, Széchenyi College, H-9026 Györ, Hungary (e-mail: benyo@mit.bme.hu).

Publisher Item Identifier S 1089-7771(02)02012-5. role: open architecture and standard information interfaces. Today the majority of applied patient monitors are built up based on a vendor-specific closed architecture without any standard communication interfaces.

The goal of our research was to establish a patient monitoring system with the following key features:

1) open architecture;

2) scalability of the system;

3) expandable signal interpretation;

4) reasonable system cost.

To achieve the goals listed above, a novel approach to the patient monitoring system design is introduced: system development based on industry standard.

\section{STATE-OF-THE-ART}

Most of the existing patient monitors belong to the so-called firstgeneration systems with traditional and quite reliable signal interpretation capabilities (e.g., border value alarming, cardiac and respiratory arrhythmia detection). Decision support and interactivity-as a higher level of signal interpretation - are the features of the second- and third-generation monitors [4]. These systems provide more advanced, usually knowledge-based signal interpretation.

From an architectural point of view, many existing systems support the networking of bedside monitors to a central monitor via a vendorspecific communication interface. The signal interpretation features of the central monitors are usually more advanced than the bedside monitors. However, there are several standards for transmitting various types of medical information [5]. The medical information bus (MIB) is the only existing standard that deals with the low-level communication technology between bedside medical devices [6]. Although the MIB draft specifies all the seven open systems interconnection protocol layers, the standard is currently ratified for the lower layers only. MIB was designed in an object-oriented way, considering the most important requirements on bedside networks: plug-and-play and fast reconfiguration, ease-of-use, reliable fault diagnostic, and patient safety. The whole MIB standard is rather complex, and because only some parts were ratified, vendors limit their efforts in implementing MIB-compatible devices [5].

\section{INDUSTRY STANDARD-BASED MONITORING SYSTEM}

\section{A. Overview}

To meet the requirements of an open architecture and scalable system, we made following design considerations [7]:

1) use of only standard, off-the-shelf components and solutions (both hardware and software);

2) vendor-independent open communication standard as bedside network;

3) standard analog input signal ranges.

The overview of the developed patient monitoring system is illustrated in Fig. 1. The components of the system are briefly detailed in the following sections.

\section{B. Bedside Network}

Several industrial communication standards were developed and approved during the last ten years. These so-called fieldbus systems 


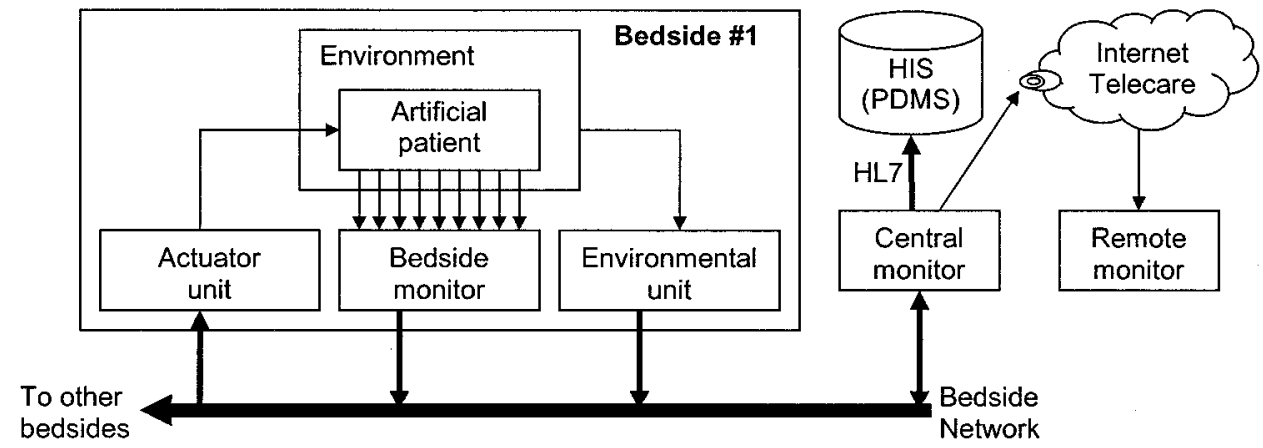

Fig. 1. Structure of the open architecture patient monitoring system.

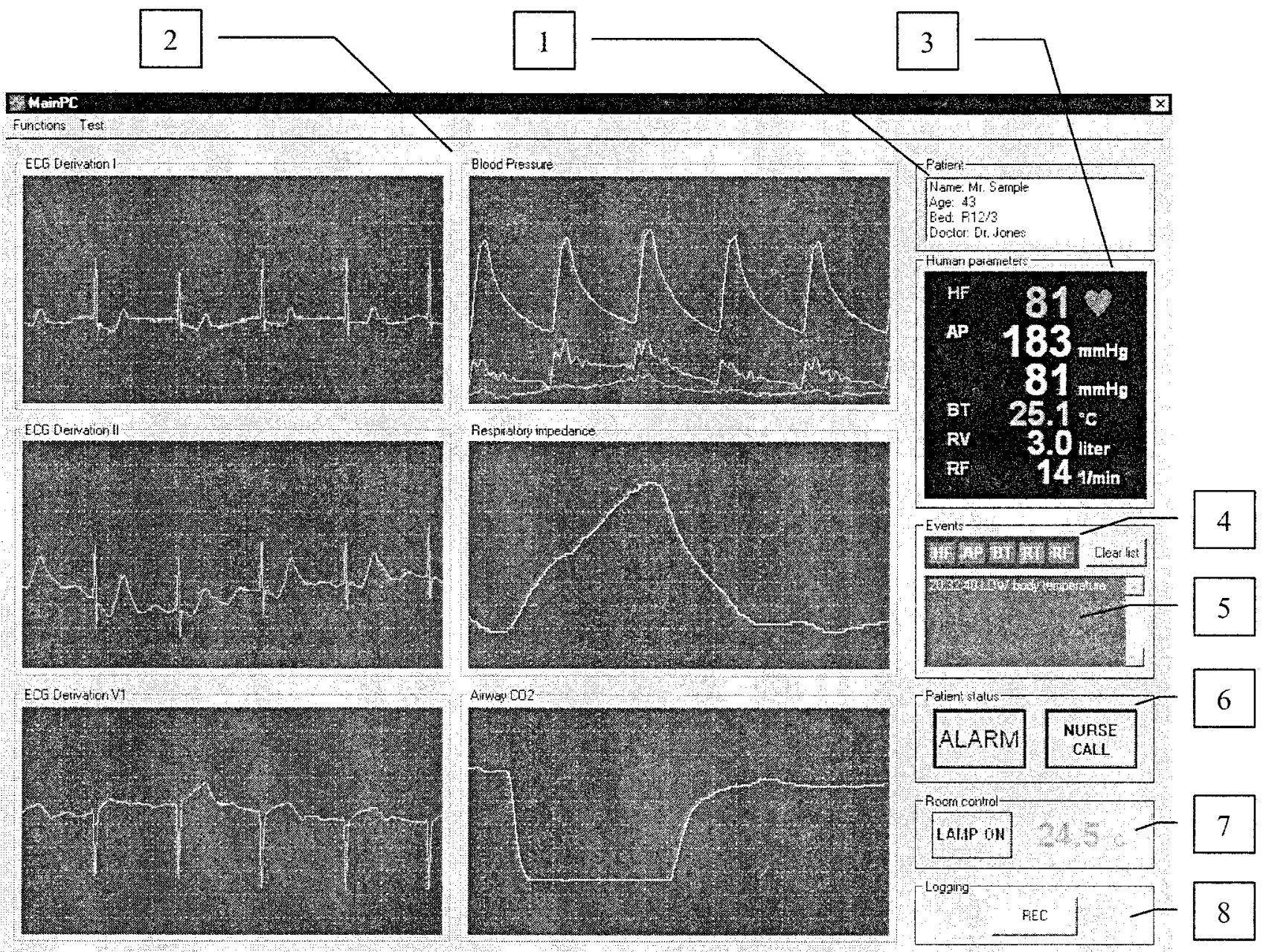

Fig. 2. The central monitor's screen. 1) bedside information; 2) signal windows; 3) HF-heart rate, AP-arterial blood pressure, BT-body temperature, $\mathrm{RV}$-respiration volume, RF-respiration rate; 4) alarm indicators; 5) event log; 6) master alarm and nurse call indicators; 7) room temperature and room light indicators; and 8) button for start/stop recording of all signals.

define communication protocols between industrial devices for process control, data acquisition, and data processing. The standards provide reliable and deterministic communication with exact timings. Additionally, the fieldbus systems have advanced features such as open structure, plug-and-play components, easy installation, and low maintenance cost.

The idea of implementing a fieldbus-based medical communication system originated in the fact that many requirements of the medical bedside communication system are met by the fieldbus systems (i.e., deterministic, time-critical, robust, redundant, and high-speed cyclical data transmission).

Our choice was the Profibus DP fieldbus standard, which was designed for cyclical data exchange at very strict timings and high data transmission speeds [8]. The devices can be interconnected using a two-wire cable on a bus topology, supporting interconnection redundancy. The bedside communication network was realized with a single- 
master Profibus DP network at $1.5-\mathrm{Mb} / \mathrm{s}$ transmission speed. The network interconnects the central monitor (master) and the bedside monitors (slaves).

\section{Artificial Patient}

The development of a monitoring system requires an appropriate signal source. In reality, the vital signals originate from human patients and are measured with electrodes or catheters. The development of a patient monitor requires a reproducible and easy-to-control signal source, which is not the case with a human patient.

Therefore, an artificial patient was designed and implemented in order to generate the required vital signals. A desktop PC was used with a 12-channel digital-to-analog converter (DAC) slot card (Advantech PCL-727, Advantech Co., Ltd., Irvine, CA) with 12-bit resolution and $0-5 \mathrm{~V}$ output signal range. With this solution, the interfacing problem of low-level physiological signals and the strict safety standards could be avoided.

The signals were originated from a multichannel reference signal database [9]. The artificial patient generates the following vital signals:

1) 1, 2-, or 3-channel electrocardiogram (ECG) (depending on the database);

2) noninvasive arterial blood pressure;

3) abdominal respiration or nasal airflow;

4) body temperature and, depending on the database, optionally:

a) pulmonary and central venous blood pressure;

b) $\mathrm{SpO} 2\left(\mathrm{O}_{2}\right.$ saturation $)$ and $\mathrm{PCO} 2\left(\mathrm{CO}_{2}\right.$ pressure $)$.

\section{Bedside Monitor}

This bedside monitor is responsible for the bedside acquisition and display of the vital signals of a patient. The device has a bedside network connection to the central monitor (see Fig. 2). The bedside monitor was realized with an 80386-based single-board industrial panel PC (PPC-55S, Advantech). The generated vital signals were measured by an eight-channel analog-to-digital converter card at 12-bit resolution. The sampling rate was $100 \mathrm{~Hz}$ in the case of the ECG and blood pressure signals and $50 \mathrm{~Hz}$ in the case of the other signals.

The bedside monitor is capable of some simple signal interpretation (limit value detection) and derives the following parameters from the measured primary vital signals:

1) heart rate (number of heart beats per minute, pulse);

2) end-tidal lung volume (liters per minute);

3) respiration rate (number of breaths per minute);

4) systolic and diastolic arterial blood pressure values $(\mathrm{mmHg})$.

As the selected industrial panel PC had no direct fieldbus communication interface, we used an external fieldbus link module, which can act as a gateway between the Profibus DP and the RS232 serial port of the panel PC [10].

\section{E. Central Monitor}

The central monitor is a desktop PC equipped with a Profibus master slot card (DFProfi PCI, Comsoft GmbH, Karlsruhe, Germany). The central monitor provides the functions as follows:

1) real-time reception of bedside data of up to 16 patients;

2) real-time display of selected bedside data;

3) signal interpretation and alarming with event logging;

4) automatic storing of all received bedside data using a 24-h ring buffer in a standard database format;

5) signal interpretation of the central monitor provided through standard software interfaces (dynamic link library and Microsoft ActiveX), which allows flexible upgrade of the functionality;

6) HIS/PDMS client (under development);

7) telecare (remote) monitoring host using Microsoft ActiveX and Wireless Application Protocol.

\section{F. Environmental and Actuator Units}

Two Profibus DP-connectable digital and analog input-output (I/O) units (RIO DPs of SAIA-Burgess Co., Murten, Switzerland) measure signals of the environment and provide outputs:

1) room temperature (input, $0-50{ }^{\circ} \mathrm{C}, 12$ bit);

2) nurse call button (discrete input);

3) room lamp (discrete output);

4) actuating signal of a simulated infusion pump.

\section{RESULTS}

The prototype of the remote patient monitoring system was implemented in the Biomedical Engineering Laboratory at the Department of Control Engineering and Information Technology, Budapest University of Technology and Economics. The current installation consists of one bedside (including a bedside monitor, an environmental unit, and a rudimentary actuator unit) and a central monitor. The devices are networked via the Profibus DP industry standard fieldbus.

The bedside monitor transmits both the primary and the derived signals in real time to the central monitor. The cyclical data transmission is scheduled by the fieldbus master (central monitor) in 100-ms cycles. The environmental and actuator signals are exchanged with the corresponding I/O units using a 1000-ms polling cycle.

The signal interpretation features of the complete system can be divided into two stages:

1) bedside monitor: simple signal failure and signal threshold detection;

2) central monitor: threshold value detection, detection of cardiac arrhythmias (classification of normal, ventricular, paced, and fusioned beats, detection of bigeminy and fibrillation), and apnea detection based on the respiration signal.

The crucial points of the system performance are the capacity of the used bedside network and the robust handling of various network failures. The $1.5 \mathrm{Mb} / \mathrm{s}$ transmission speed of the single-master bedside Profibus DP network allows approximately 100 kbytes of user data transmission per second. The real-time transmission of the measured vital and environmental signals of one bedside requires less than $5 \mathrm{kB} / \mathrm{s}$. Therefore, the fieldbus network is able to interconnect the central monitor with up to 16 bedside monitors. The evaluation and verification of the system was carried by using an additional desktop computer as a fieldbus data traffic and bus load monitor, which was equipped with a Profibus Class 2 master slot card (DFProfi ISA, Comsoft).

The present installation with one bedside results in $4 \%$ network traffic load measured by the bus load monitor. The network data traffic of the other 15 bedsides was simulated by a Profibus bus load simulator device [11]. In this case, the total bus load (altogether 16 bedsides) increased up to $75 \%$. The bus-load simulator also proved to be helpful to simulate various network failures, e.g., data losses due to device malfunctions and fieldbus errors. The data transmission performance of the still active bedside monitors was not affected by the failed bedside monitors as far as the central monitor remained active. This result was provided by the underlying Profibus DP communication technology, which fulfilled the preliminary expectations.

\section{CONCLUSION}

A new and alternative way for the development of patient monitoring systems has been proposed: industry-standard system components and fieldbus communication technology. The novel approach of using industry-standard off-the-shelf solutions resulted in a scalable design at a reasonable development cost and increased the flexibility and maintainability of the patient monitoring system. The main benefit of the system from the signal interpretation point of view is in the design of 
the central monitor. The central monitor allows the real-time access of bedside data via standard software interfaces, so the upgrade of the signal interpretation layer can be realized without affecting the other components.

The feasibility of proposed system was verified by the implementation of a demonstrator system. The performance evaluation and system design verification have shown that the system is capable of robust real-time handling of up to 16 bedsides. Although the system design neglects some of the real aspects of patient monitoring (i.e., the real vital signal properties and ranges, strict standards for patient safety), it provides a fair and flexible demonstration of the complex problem of patient monitoring.

\section{ACKNOWLEDGMENT}

The authors would like to thank T. Micsik, M.D., for his guidance and support.

\section{REFERENCES}

[1] J. H. van Bemmel and M. A. Musen, Handbook of Medical Informatics. Houten/Diegem, Germany: Springer, 1997.
[2] K. Wang, I. Kohane, K. L. Bradshaw, and J. Fackler. (1996) A real-time patient monitoring system on the WWW. [Online]. Available: http://www.emrs.org/publications/amia_icu.html

[3] P. R. Norris, B. M. Dawant, and A. Geissbuhler. (1997) Web-based integration and annotation in the intensive care unit. [Online]. Available: http://simon.project.vanderbilt.edu/pub/amia97

[4] E. Coiera, "Automated signal interpretation," Monitor. Anesth. Intensive Care, pp. 32-42, 1994.

[5] Hewlett Packard Company, "Communication standards in the clinical setting: A closer look at the details," Technology White Paper, Pub. 5967-5483E, 1998.

[6] IEEE 1073 Medical Information Bus. [Online]. Available: http://grouper.ieee.org/groups/mib

[7] Z. Benyó, B. Benyó, and P. Várady, "Patient monitoring on industry standard fieldbus," in Proc. 1st Joint BMES/EMBS Conf., Atlanta, GA, 1999, p. 704

[8] European Open Fieldbus Standard, European Standard EN50170, 1996.

[9] G. B. Moody and R. G. Mark, "A database to support development and evaluation of intelligent intensive care monitoring," Comput. Cardiol., vol. 23, pp. 657-660, 1996.

[10] P. Várady, An SPC3-Based Universal Serial Fieldbus Link Unit. User's Guide. Budapest Univ. Technol. Econ., Hungary: Dept. Control Eng. Inform. Technol., 1999.

[11] M. Heinz, "Buslastemulator für profibus," diploma thesis, Institute of Computer Technology, Technical Univ. of Vienna, Austria, 1996. 